\title{
An Intelligent IOT Mechanism for Collision Detection and Prevention at Railway Level Crossing
}

\author{
Raja Sekhar Kummari, Phani Sridhar Addepalli, Krishna Mohana Tenneti
}

\begin{abstract}
With the rapid growth of things connected to Internet leading to the massive scale on increase of devices for automation and control. An explosive demand adds the strength to ubiquitous computation and the device interaction over cyber physical systems. This commendable support is taken in to practice for giving an automated IOT solution for the railway level crossing problems faced across the countries. Especially in populated countries like India the size of traffic on roads and railways is happening in a short time at a great rate. The situation is always alarming at the exchange of transport between these mechanisms at a common point called railway level crossing. In this paper we are providing a novel, cost effective and intelligent IOT solution using Raspberry Pi outfitted with sensors, RFID tags and load cell to predict the arrival or crossing of trains, also controlling the gates to get rid the problems arising manually by restricting the vehicles not to face the disasters. It enhance the effectiveness, trustworthy of the railways in the community of passengers.
\end{abstract}

Index Terms: CPS, Load Cell, MQTT, RFID, Sensors, Ubiquitous Computing, Raspberry Pi.

\section{INTRODUCTION}

Railway is considered as one of the cheapest and safest mode of transport worldwide. Therefore, safety is the crucial aspect in railway operation everywhere. With the ever increasing population, the railway industry always encounters separate problems due to technical and human errors, especially at level crossings. The Level crossing is a cross-sectional area where rail track and roadway intersect each other. In most cases, level crossing remains unattended mostly in rural and remote areas. Hence, such intersection requires constant human coordination and monitoring on regular basis. The level crossing is divided into two types namely manned and unmanned. Almost every year there are accidents/collisions reported at level crossing. Nearly 5,800 unmanned railway crossings are a key cause of rail accidents in India. As per a report 109 rail accidents have occurred at unmanned crossings during the past years. As per the report from Times of India $66 \%$ of accident fatalities occur at level crossings due to carelessness of road users and the gatekeepers. Of all the road infrastructures, level crossing is considered as one of the most dangerous spot. Actually, 29\% of mishap happens in railroad framework occur at level

intersection. Much of the time, mishaps occurred because of lack of regard of street clients and vehicle administrators towards detached street signs or presumably due to awful climate conditions. From the investigation of the reason for the mishap Indian Railways found was that of the considerable number of mishaps $87.78 \%$ were because of the human disappointment. Subsequently, there is a critical need to dispense with these components. To wipe out these variables we have to cause things to speak with one another and consequently the M2M innovation goes to the image [1].

Indian Railways was formulated for a period of ten years (2003-2013) entailing multi-pronged strategy, laying emphasis on prevention by reducing human dependence and mitigation of consequential effect in case of an accident. The Corporate Safety Plan envisages reduction of accidents on IR by the year 2012-13, substantially by bringing down the accident rate and is better than many of the advance Railways of the world.

The Road Traffic crosses the Railway Track either on "Grade Separated Crossing" (different Levels of rail and road) or at "Level

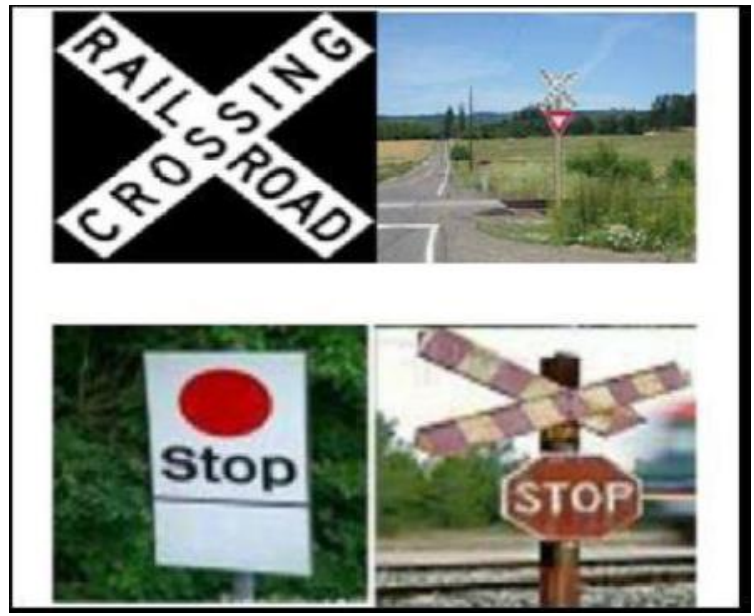

Crossing" (Road and rail are at same levels). The level crossings are made to facilitate the smooth running of traffic in a regulated manner governed by specific rules and conditions. 
Figure 1: Signs and Scenario at LC.

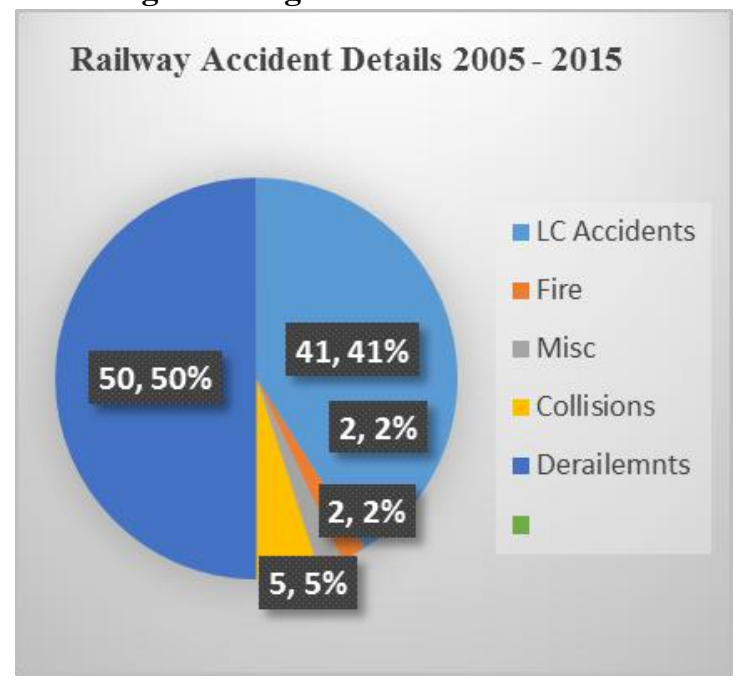

As on recorded information, there are a total of 31846 level intersections spreading over more than 64460 course kilometers for example normal of 49 level intersections for every 100 kilometers in Indian Railways. Out of which 13,530 Figure 2: Rail mishaps because of numerous reasons.are unmanned and 18,316 are kept an eye on. Out of 18316 kept an eye on level intersection 9,978(54\%) are interlocked for example ensured by a Gate Signal as an extra Layer of Safety. The period from 2007-08 to 2011-12 uncovers that an enormous piece of 779 passings (58\%) and 670 wounds $(27.5 \%)$ were because of unmanned level crossing accidents attributed to the negligence of road users and it constituted $36.4 \%$ of the total train accidents . Manned level crossing accidents which are $4.5 \%$ of total tally resulted in $4.8 \%$ deaths and $5.5 \%$ injuries (refer Figure 2 and 3).

The Level Crossings are vulnerable to the extent that it is in charge of $41 \%$ of mishaps, $63 \%$ passings and $33 \%$ wounds over Indian Railways. The essential drivers of mishaps at unmanned level intersections incorporate disappointment of street clients is misfortune to cross level intersections even with a moving toward train.

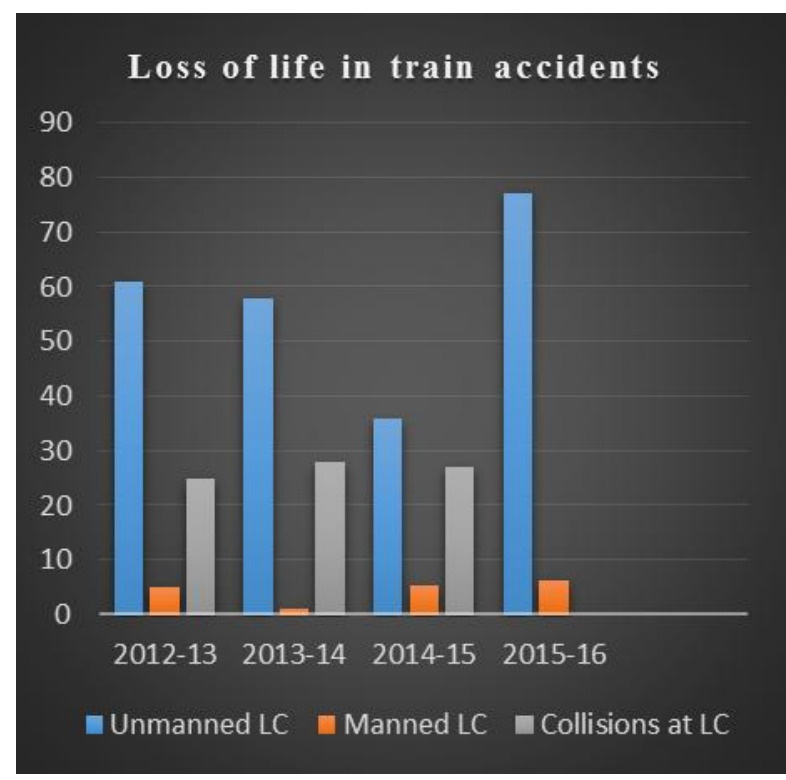

Figure 3: Cause of death at LC.

Street vehicles getting slowed down at the areas, rash driving of un-authorized tractor drivers and so forth. Mishaps at level intersections happen fundamentally in light of the fact that the street clients don't regard the option to proceed of railroads. It is seen that the street vehicle drivers misconstrue the speed of trains because of the way that human response time is 2.5 seconds which is only enough to arrange the reflexes against speed of $60-70 \mathrm{kmph}$. Anyway prepares on Indian railroads are utilizing at around 100-120 kmph for which the response time is lacking. Street clients keep on intersection the tracks regardless of whether the train is unmistakable and moving toward making driving level intersection mishaps. The issues of mobility and accident prevention at level crossings can best be addressed by joint efforts of all concerned - Central Government, State Government, Municipalities, NGOs, educational institutions and private sectors etc.

\section{SAFETY INFORMATION MANAGEMENT SYSTEM}

An electronic framework for generally Safety Management of Indian Railway has been created in which one of the modules is over level intersection. This Level Crossing Management System screens the information of level intersection by relegating a novel to each level intersection. The Unique ID further associates to every one of the advancements like example of Traffic, signage, condition, up degree works, mishap subtleties connected with satellite symbolism.

CORRELATION OF TRAFFIC VEHICLE UNIT AND NUMBER OF LEVEL CROSSING:

\section{TRAFFIC VEHICLE UNIT (TVU):}

The vulnerability of a level crossing is measured in terms of TVU which is obtained by multiplying the number of trains with the number of road vehicles passing over the level crossing in 24 hours, where, train, road vehicle, bullock carts and tongas are considered one units and cycle rickshaw / Auto rickshaw half unit. A periodical census of all level crossings is done once every three years for seven days.

\section{UNMANNED LEVEL CROSSING:}

There are complete 13530 unmanned level intersections. It is apparent that on the off chance that we dispense with 1447 (13530-12083) unmanned level intersection, which is about $11 \%$ of complete Unmanned LC then we can take out all Unmanned LC over 3000 TVU. This will diminish the ACCIDENT RISK by $44 \%$ of all out level intersection mishaps.

\section{MANNED LEVEL CROSSING:}

There are all out 18416 Manned Level Crossing. From the investigation it very well may be seen that if 2316 (18416-16100) kept an eye on level intersection, which is about $13 \%$ of absolute Manned LC, at that point we can dispense with all Manned LC above 0.1 Million TVU. In this manner, by developing 2316 ROB (Road Over Bridges) we can get free of High TVU Manned Level Crossings. Machine to machine (normally shortened as M2M) alludes to coordinate correspondence between gadgets utilizing any 
interchanges channel, including wired and remote Machine to machine correspondence can incorporate mechanical instrumentation, empowering a sensor or meter to convey the data it records, (for example, temperature, stock level, and so on.) to application programming that can utilize it (for instance, altering a modern procedure dependent on temperature or setting requests to renew stock). Such correspondence was initially practiced by having a remote system of machines hand-off data back to a focal center point for investigation, which would then be rerouted into a framework like a PC.

\section{II.RELATED WORK}

Extensive work has been envisaged in the past to reduce the causalities, collisions, accidents and other up hailed disaster happened in the past. At present major accidents are due to possibility in absence of railway gates at level crossing and the negligence of operators, parallax disturbances even though there are railway gates. In a closed system of Indian railways the feedback control is more important as the way of reducing the collisions and manual errors are utmost necessary.

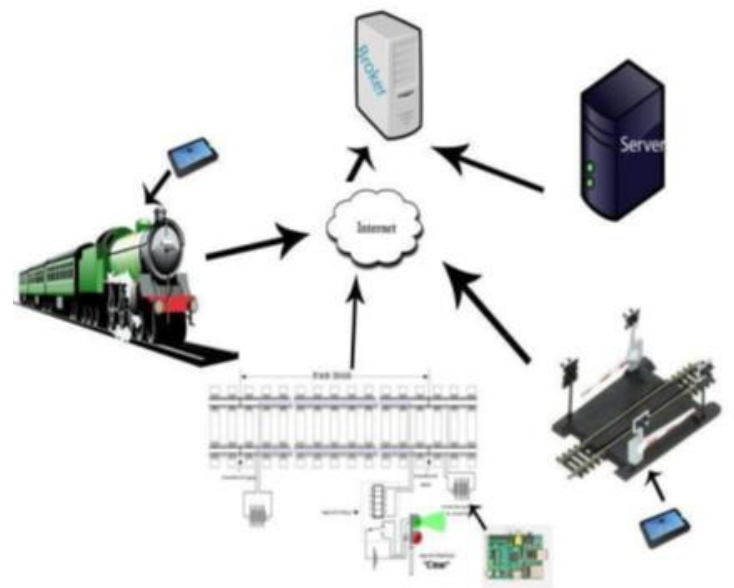

Figure 4: Prototype self-controllable LC

This made the signalling and reporting of level crossing with the number of trains passing over road or bridge is treated necessary but not sufficient. In a recent work trains are fitted with the RFID tags that signals the transceiver to arrange the precautionary action at the need of the hour (Figure 4). This is called Self Accelerated Control Action (SACA) [3]. In this marvel the self-controllable activity is started to control the track snatching limitation of vehicles and trespassers. It is necessitated that the sign and point to be interlocked so that at whatever point a train is overwhelming at stations a sheltered section will be made accessible for trains. Presently as these are interlocked there are not many interlocked level intersections which at some separation gets interlocked at whatever point train goes through that and thus the level intersections gets naturally shut these level intersection entryways are called Interlocked doors which are chiefly found in the urban pieces of the nation. Not all doors are interlocked entryways and subsequently numerous entryways are physically controlled. These doors are enormously subject to the human correspondence as the individual who is keeping an eye on the entryway is educated by means of phone. At unmanned level intersections there are no boundaries and lights for the data of the train entry, just indication of the level intersection is made accessible at 200 meter separation [2].

In another work, Highway-rail grade crossing collisions are a significant safety concern around the world. Road rail collisions have a high risk of injuries and fatalities. To mitigate that risk, safety countermeasures for both active and passive crossings have been implemented. Leveraging the latest developments in connected vehicle (CV) technologies, $\mathrm{CV}$-based warning systems perform well in safety applications for roadway networks. Few works have been developed to focus on safety improvements specifically for road rail level crossings. Some work proposes a novel active warning system that was created with readily available $\mathrm{CV}$ technologies and devices [4]. A crossing risk assessment model was developed and evaluated in simulation and field applications. When road users are in great risk of a collision, the warning system sends out auditory and visual alerts with estimated time of safety reaction.

\section{METHODOLOGY}

The Taxonomy of device communication has taken a formal presentation called Machine to Machine communication (M2M). The connected things are straight parameters for action to take and react on the machine that is in need of control of necessary reactions. Multiple suggestions along with extension is made in technology in due vision of vehicles to be connected and things to be connected over internet leading to a new era of device communication and things connectivity called Internet of Things (IoT) [6]. In this paper an intelligent prediction and detection mechanism using Internet of Things is provided as a solution for the long trending high escalating collision problems at the stringent level crossing in railways [8].

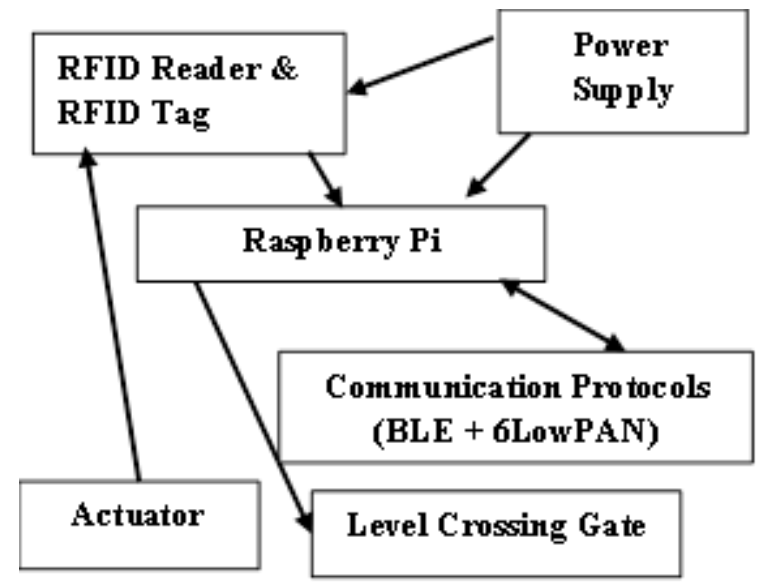

Figure 5: System Implementation with Protocols 
The system incorporates RFID Readers and Tags, used to detect the arrival and departure of the train at level crossing. Raspberry $\mathrm{Pi}$ is used as an Internet of Things controller to control the opening and closing of the gate with the help of actuator. The system comprises of RPi, Servo Motors, RFID Readers and Tags. The proposed system attempts to develop a system that automates the gate operations with effectively without any defects [7] departure of trains at the railway level crossing and a servo motor to control the opening and closing of gate [5]. The train is detected when the RFID Reader reads the Head Tag attached to the train and the automatic alert system becomes active and alerts the commuters and the station master by blowing n alarm (Figure 5).

Later, when the RFID Reader reads the Tail Tag the Signal System gets active and the Orange Signal turns to Red and the gate is kept closed at cross section within predefined time interval. Signals generated by RFID Reader and tags (Figure 6 and 7) are sent to the Node MCU and this will configure the operation of the Servo Motor and it will rotate as required requirement (clockwise or anticlockwise).

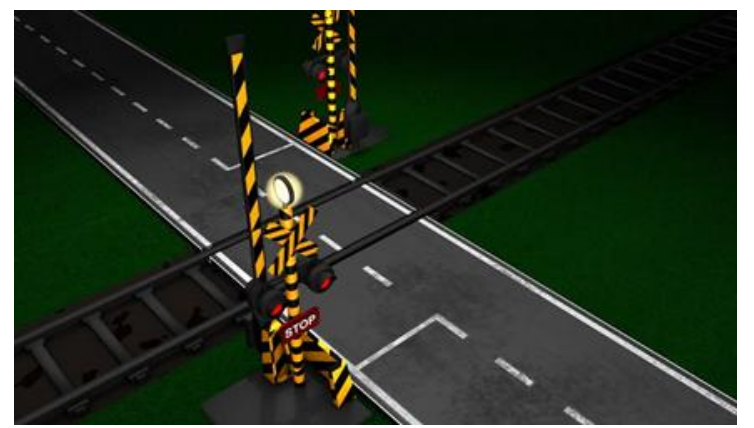

Figure 6: Image of RFID tag Installation at LC.

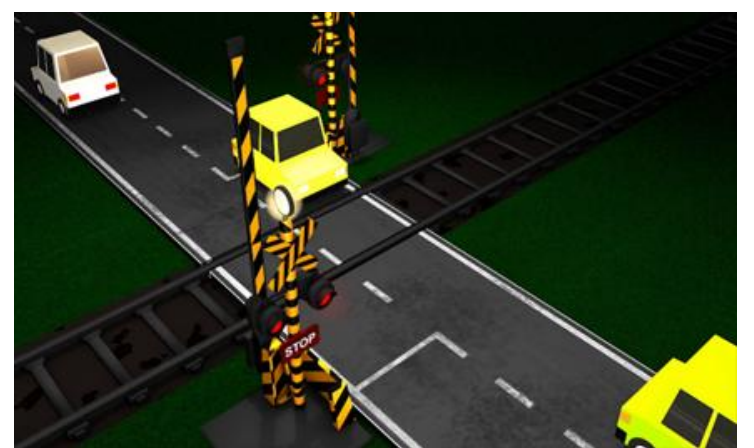

Figure 7: Image of RFID tag Installation at LC.

Unauthorized objects on the track are detected using Load Cell placed at the crossing level. The signals generated of unauthorized objects on the track, closing, and opening of the gate is transmitted to the central control room via wireless communication protocol (BLE). It also takes the support of network communication for understanding the low power loss networks. As it is highly framed that the loss of data is not at all expected for the gate control at level crossing it is always suggested to take non data loss protocols like 6LoWAN.As the semi-automated existing systems are creating vulnerability at the cross sections of rail road bisection, the bunch of lives are being lost. The reliability and response time is utmost priority while the data transfer and reaction time will be take place in small time.
It uses RFID Tags and Readers to detect the arrival and

The data always reaches the controller as a continuous flow from the sensors/ tags. When a train is passing near by the installed load cell then the cell leaves a control data to the RFID tags in turn detects the object moving towards the railway gate. The transceivers installed will operate by closing the railway gate in advance. The prior time of action is much more than proportional to the speed of the train reaching the level crossing. There are multiple restrictions and limitations to the time frame fixing for the operation of level crossing gates.

\section{IV.RESULTS AND DISCUSSIONS}

This section gives the abstract information of results obtained in a statistical form with respect to the response time of gate closing and opening in view of wireless data communication protocols (BLE) as well as network communication protocol (6LoWPAN).

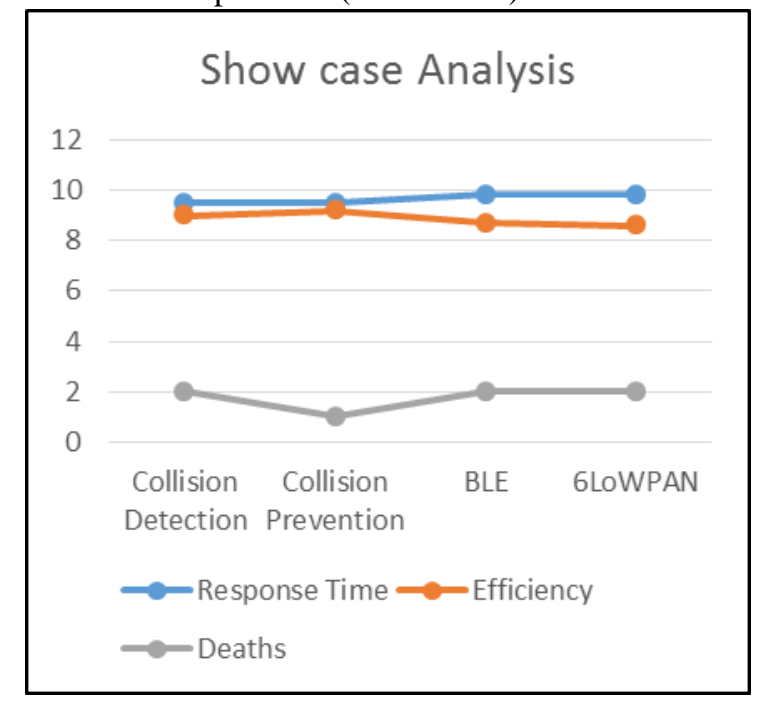

A web application along with the data sense mechanism can also be suggested to travel with theefficiency at higher levels. The IoT solution provided here will optimizes the response time along with the reaction time. An extension can also be expected here by communication to the station master or operator at the nearby railway cabin to respond if there is any risk.

\section{CONCLUSION AND FUTURE SCOPE}

A further work can be possible to generate the short message service alerts to the passengers about to board the train in the railway station near the level crossing. Also the same alert can be generated to the passengers travelling in the train about the expected arrival of the station nearby. The same procedure can be developed and in variety of technologies like NodeRed to optimize the socket connectivity issues and making it more easy. The much more awaited design technology is also possible for the design constraints available at the expectation to Mobile phones, social networking websites and whatsapp messenger too. A control action is also possible by selective and efficient cloud data storage and responses to the system. 


\section{REFERENCES}

1. E. Arunakanthi and H. SudarsanaRao, "Effects of Sulphuric Acid in Mixing and Curing Water on Strength of High Performance Phosphogypsum Concrete", IJSTE International Journal of Science Technology \& Engineering, Volume 2,Issue 08, February 2016.

2. V. Ramasamy, "Compressive Strength and Durability properties of Rice Husk Ash Concrete KSCE Journal of Civil Engineering, Volume 16,Issue 01, pp 93-102, January 2012.

3. Chia khai tee, A project submitted to civil engineering and earth resources university Malaysia Pahang under title "performance of spent bleaching earth as cement replacement in concrete", Nov 2010

4. Linaylchongann, A project submitted to civil Engineering and Earth Resources University Malaysia Pahang and under title "strength of concrete with spent bleaching earth as cement replacement", Nov 2010

5. Wachira J, Muthengiawa-Thiong'oJ.Karanja, Gerald K.Muthakia,"Spent Bleaching Earth as a Pozzolanic Material" African Journals(AJOL),Vol.2,Issue 01,2005.

6. R.Ilangovana, N.Mahendrana and K. Nagamanib, "Stength and durability properties of concrete containing quarry rock dust as fine aggregate", ARPN Journal of Engineering and Applied Sciences, Volume 3, Issue 05, October 2008, ISSN 1819-6608, ISSN (online): 2349-784X.

7. Ogork, E.N; Aboshio, A. and Balami, D.A, "Durability Assessment of Concrete Made with Rice Husk Ash as Admixture", Journal of Engineering and Technology (JET), Volume 05,Issue 01, February 2010.

8. Arvind Kumar, Amit Kumar Tomar ,ShravanKishor Gupta, Ankit Kumar, "Replacement of Cement in Concrete with Rice Husk Ash", SSRG International Journal of Civil Engineering (SSRG-IJCE), Volume 3,Issue 07, July 2016.

9. SohKheangLoh, StephenJames, MuzzamilNgatiman, KahYeinCheong, Yuen MayChoo, WengSoonLim, "Enhancement of palm oil refinery waste - Spent bleaching earth (SBE) into bio organic fertilizer and their effects on crop biomass growth", ELSEVIER, Volume 49,PP 775-781, August 2013. 